\title{
RADIO FREQUENCY AND CHANNEL INVESTIGATION USING SOFTWARE-DEFINED RADIO IN MATLAB AND SIMULINK ENVIRONMENT
}

\author{
S. O. Ugwuanyi ${ }^{1,}{ }^{*}$ and M. A. Ahaneku' ${ }^{2}$

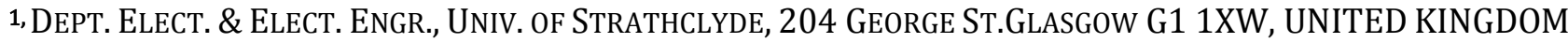 \\ 2, DEPARTMENT OF ELECTRONIC ENGR., UNIVERSITY OF NigERIA, NSUKKA, ENUGU STATE, NIGERIA \\ E-mail addresses: ${ }^{1}$ stephen.ugwuanyi@strath.ac.uk, ${ }^{2}$ mamilus.ahaneku@unn.edu.ng
}

\begin{abstract}
This paper is a four section investigation of various communication principles to demonstrate the capabilities of using $M A T L A B$ and Software Defined Radio (RTL-SDR R820T) to receive, decode, analyse and resample a radio frequency signal. A novel MATLAB model system is developed to identify signals based on the signal characteristics in the spectrum analysers. The bandwidth, frequency, modulation and demodulation techniques of the signal were also identified. The section two is a design developed around multiplex and down-conversion to baseband Inphase/Quadrature phase (IQ) for multiple channel Frequency Modulated (FM) and Amplitude Modulated Double Sideband Transmitted Carrier (AM-DSB-TC) signal received. The functional description of sampling, decimation and interpolation form part of the contribution of this paper. The final section of the paper presents a short research on the potential benefits and a survey into the future of Software Defined Radio (SDR).
\end{abstract}

Keywords: Modulation, Decimation, Demodulation, Interpolation, Frequency, MATLAB/Simulink

\section{INTRODUCTION}

Software Defined Radio (SDR) is a computer based software transceiver scanner that makes use of terrestrial standards in the modified Digital Video Broadcasting Television (DVB-T) principles [1]. This is achieved through compression and conversion of signal's In-phase/Quadrature phase (I/Q) components into a wideband software to enable high frequency reception and processing [2]. It is a paradigm shift from a traditional hardware analogue or digital based system of radio transceiver to a software based characterized by signal processing tool boxes and commands. It main function is to perform all waveform processing like modulation, channel coding, filtering on a computer system with high speed processing like interpolation, decimation, digital up and down conversion in a processor. The implication of this is that the border between the analogue and digital transceivers is being pushed closer to the radio frequency by adopting analogue to digital (ADC) and digital to analogue conversions (DAC) close to the antenna. MATLAB is one of the engineering software that have many SDR toolboxes. The major difference between SDR and other radios is that SDR is dynamic in frequency spectrum usage (see Figure 1). It has an ungraded crystal, capacitors and inductors when compared to other radio devices.

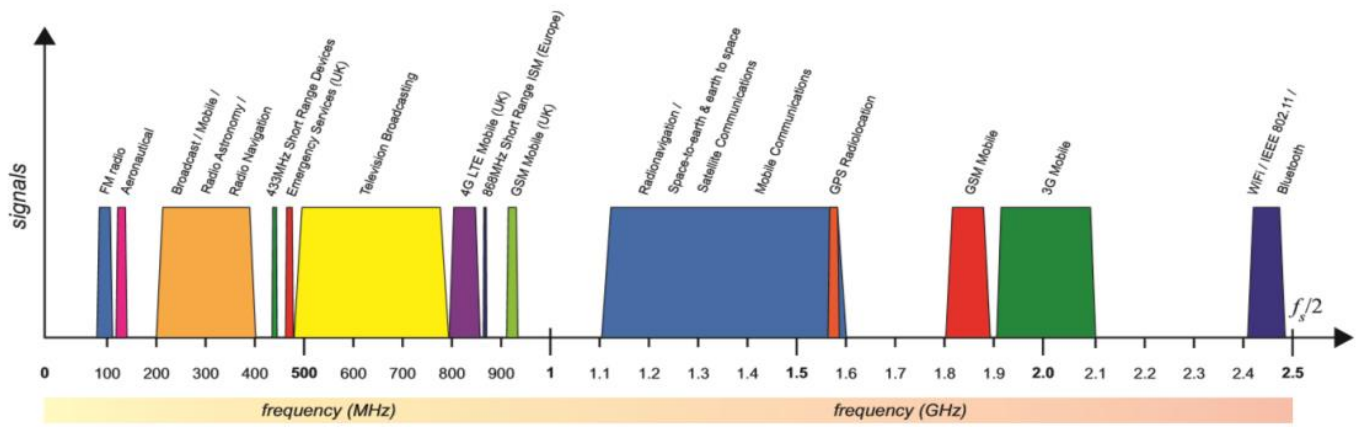

Figure 1: Frequency Spectrum [1] 
The advantages of SDR includes a compatible and flexible architecture with many software like MATLAB to various wireless standards such as frequency bands, bandwidths, waveform, and modes of operations in their configurations [3]. It leads to a better understanding of teaching and learning of abstract mathematical concepts of communication and signal processing topics like modulation and demodulation by means of simulations in Simulink. It is easier to load an appropriate program instead of building circuitry for different radio signals and protocols. It is cheap in terms of component cost because the hardware parts are now being replaced by Digital Signal Processing (DSPs) and Field-Programmable Gate Array (FPGAs) as seen in Figure 2 which is more efficient in terms of costs and efficiency. It has the advantages of remote access as with a capability to pick up multitude of frequencies ranging from $25 \mathrm{MHz}$ to $1750 \mathrm{MHz}$.

However, it tends to require more power consumption for a given function than hardware designed with optimum analogue/digital architecture partitioning. The technology of SDR design is limited by bandwidth, power and demodulation techniques allowing AM, FM, FSK, PSK, ASK, QAM radio modulation schemes internal to single system. Security, system complexity and noise are also a challenge. SDR is best suited for a system with very high speed and latest operating system. It is applied in the development of multi-standard communications systems for interoperability among users where a new modulation method, frequency range, coding scheme among others could be downloaded [4]. SDR is also applied in noncommunications areas like RADAR and RFID system where pulse schemes and RF echoes can be implemented in software like MATLAB (see Figure 3). SDR coined by J. Mitalo in 1991 according to [5] is an emerging technology that has been an active research area over the years. The development of power processors and chips has brought about the exploitation of SDR hardware platforms such as RTL. The evolution of SDR is led to a common global radio communication standard allowing reconfigurable terminals devices to adapt to different regional radio interfaces. Because of its real- time characteristics, it is becoming a major tool in industrial applications. SDR will eventually become the modern tool for wireless radio communication by integrating cognitive radio into wireless communication such as in $3 \mathrm{G}$ and $4 \mathrm{G}$ networks [2]. This will allow the creation of autonomous, intelligent, and adaptive systems where one device can communicate with other devices while maintaining a good power consumption rate and proper utilization of the bandwidth. An emerging technology of this sort could remotely be used to add receiver into a virtual instrumentation and automotive radio networks.

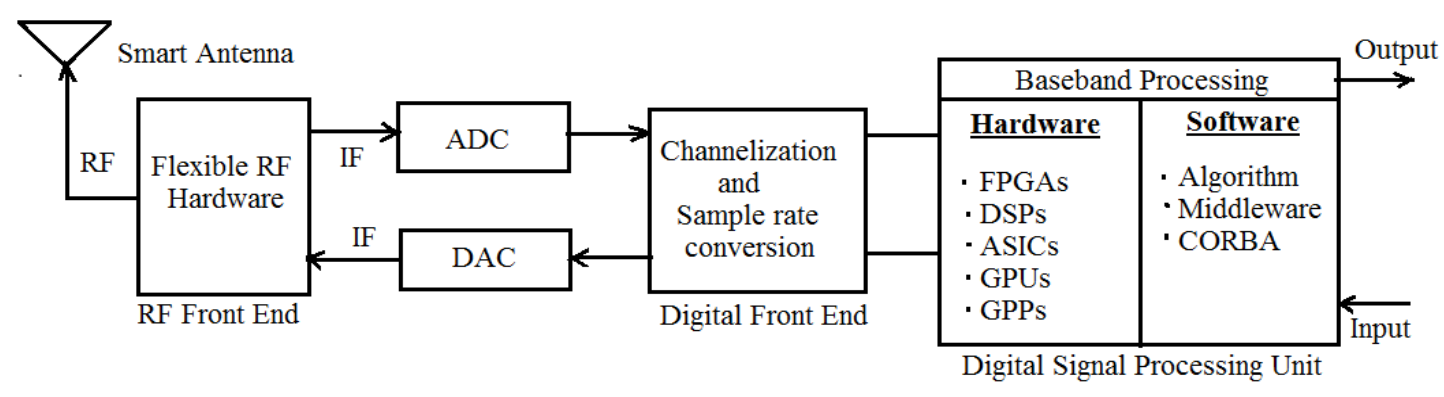

Figure 2:. Simple SDR Architecture

\section{RF Antenna}

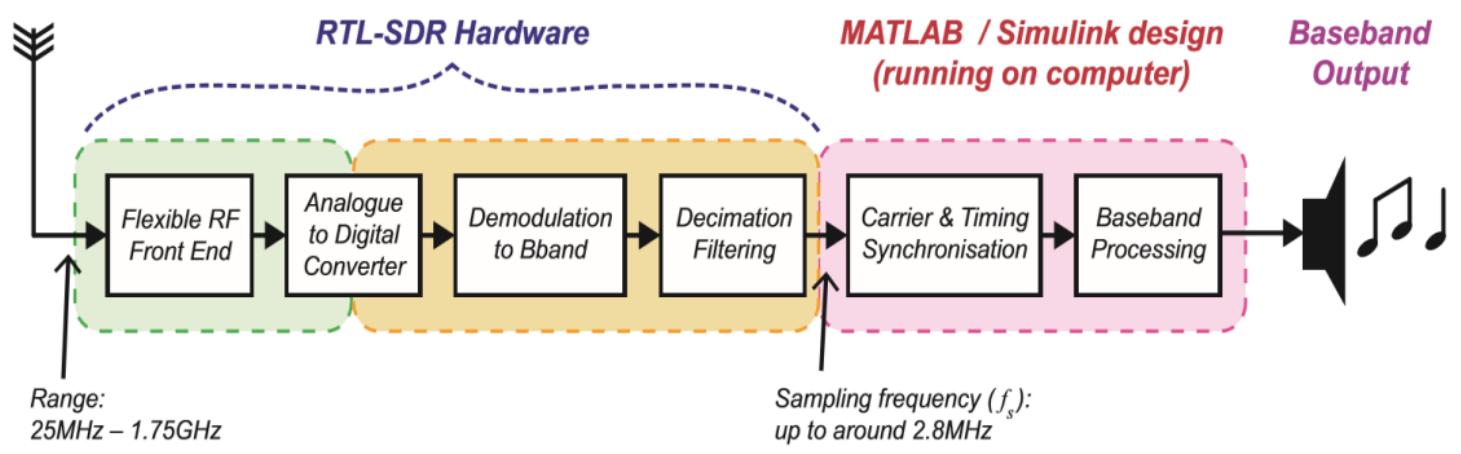

Figure 3: Block Diagram of SDR-RTL Receiver [2] 


\section{REVIEW OF RELATED LITERATURE}

Using SDR to view and analyse RF Signals in time and frequency domains and the development of DSP MATLAB enabled systems to handle the signal conversion has attracted attention in recent times. It is more difficult to perform this analysis at the physical layer DSP because of the real-time challenging concepts such as filter designs, synchronization and timing. This study system will demonstrate how SDR-RTL is used to build a complete communication system that can reduce transmitted signal payloads. SDR has been a topical research area to replace the expensive hardware electronic systems. It can be used to receive a variety of signals within $25 \mathrm{MHz}$ to $1.75 \mathrm{GHz}$. SDR as a cheap electronic device in combination with MATLAB tool boxes can handle FM reception, time/frequency domain spectral analysis and decoding of signal characteristics. The scope of this work is the investigation of signals using Software Defined Radio in MATLAB.

There are limited literatures that illustrate the capability of using RTL-SDR device for signal analysis in MATLAB. A review into the technological advancement of SDR shows a convergence of digital radios and real-time downloadable software which are responsible for wireless and internet revolution. The work of [6] states that the massive growth and convergence of the duo is now ahead of the existing technologies in the wireless industry. Many companies and research institutions have been working on the SDR for decades in an open standard. [6], identified United States Army are using SDR Joint Tactical Radio System (JTRS) in war fighters in the digital battle field environment. This work foresighted a commercial widespread applications of the technology.

SDR technology has been in its developmental stage for the past two decades. The premise on which it was built has improved significantly. SDR technology was developed to allow heterogeneous devices to communicate or even become more interactive just like the computer system. According to [7], SDR technology when properly developed will provide a path towards the realization of concepts such as reconfigurability/run-time, reconfiguration and achievement of self-governed learning radio. It will facilitate dynamic spectrum allocation, and radio access resources. Many Research and Development in the universities is ongoing to find out the best SDR architecture, supporting technologies, potentials gain in spectrum usage and the impact of SDR technology on the spectral efficiency of future communication systems. SDR is now being used in complex system application such as evaluating chips performance, digitization of Radio Frequency (RF) signals amongst others.

- $\quad$ Flexibility in spectrum usage such as trading and borrowing

- Collation services between different Radio Access Technologies (RAT)

- Distinction of services, frequency bands and interface technologies.

The major researchers include the United States Joint Tactica Radio System (JTRS) Programme, DARPA next Generation (XG) Programme. Some of the expected outcome is the dynamic reconfiguration of radio equipment, increased spectral efficiency and spectrum utilization. In [8], shared use of spectrum delivered utilization efficiency between $38 \%$ to $52 \%$ for schemes without and with spectrum borrowing respectively according to simulated exercises.

\section{RADIO FREQUENCY (RF) SIGNAL INVESTIGATION, BANDWIDTH AND MODULATION IDENTIFIER}

SDR is a wireless communication system having significant part of it defined in software instead of conventional hardware radio system. This technology came with several advantages ranging from ease of development to flexibility in reconfigurations. MATLAB is one of the tools with many support packages for SDR. To further, understand how SDR works in Simulink, recorded signals were at one stage or the other used to carry out this study. The identification of the type of radio frequency signal, the signal bandwidth, modulation and demodulation techniques in the radio frequency spectrum has always been a difficult task to achieve using SDR toolbox in Simulink. To identify the radio spectrum of the given signal,

Figure 4 consisting of two spectrum analyzers configured as Fast Fourier Transform (FFT) and waterfall of the same bandwidth were employed.

The results in

Figure 4 FFT and waterfall above shows group of burst independent signal having separation (wide band) of $200 \mathrm{kHz}$ as specified by the GSM standard for both uplink and downlink bandwidth. It is evident that the signal under study is a Global System for Mobile Communication (GSM) whose frequency of operation ranges from 900 to $960 \mathrm{MHz}$. Proper examination of the spectra shows that channels $A$ as indicated in waterfall analyser contains much powerful signals than others like channel $\mathrm{B}$ and cannot be used to determine the busty nature of the packet because it has almost a continuous transmission rate (broadcast control channel).

Vol. 37, No. 4, October, 2018 


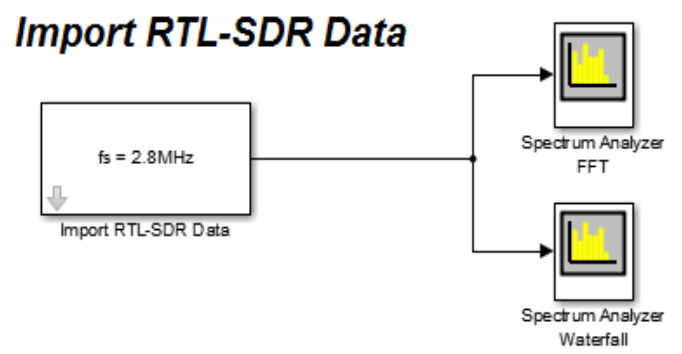

Simulation Model
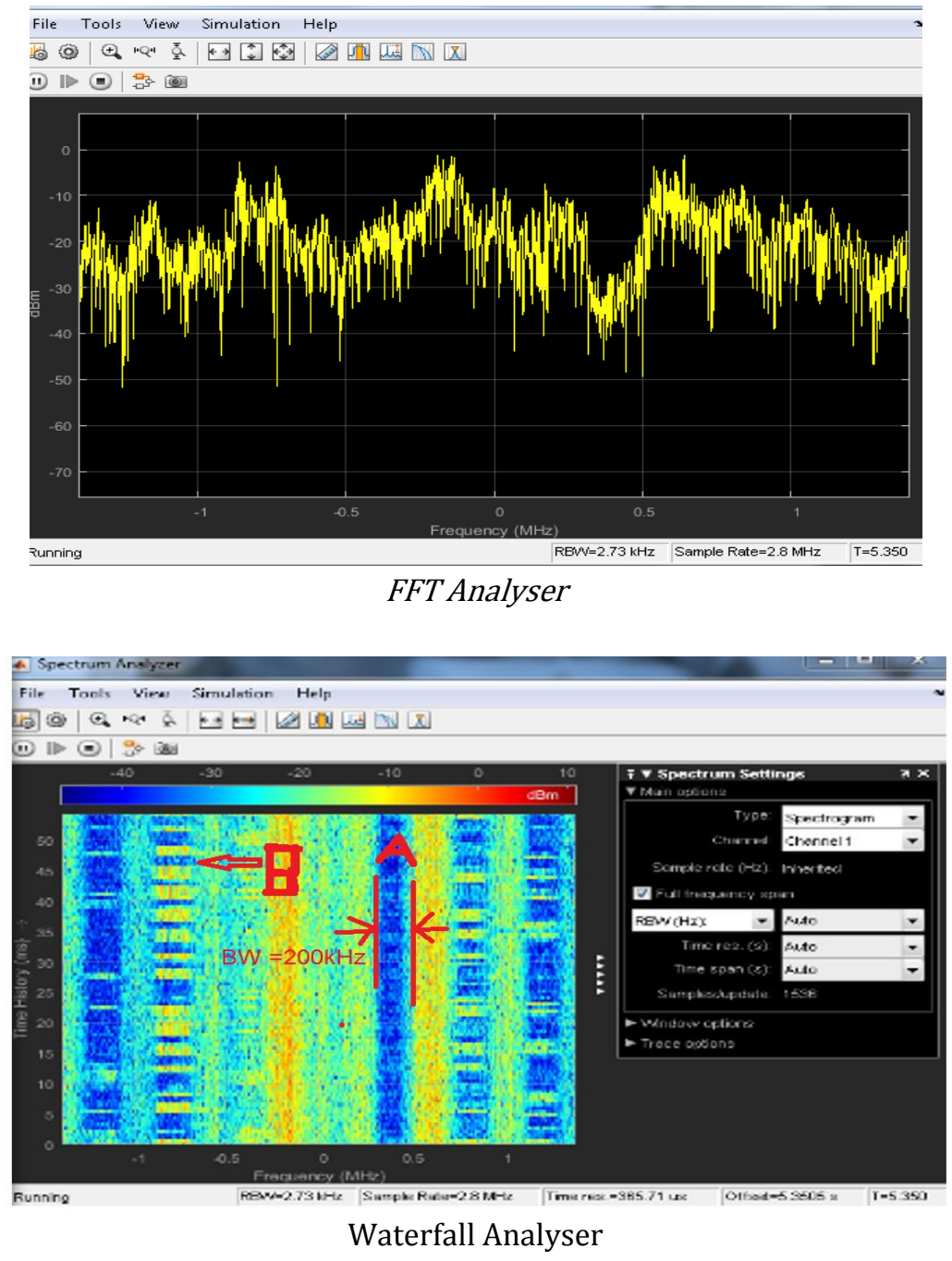

Figure 4:. Signal Investigation Model and Results

Also, a critical examination of

Figure 4 shows that Time Division Multiple Access (TDMA) is the channel access method for the shared medium. It allows many users to share the same frequency channel by dividing the signal into different timeslot [1]. The period of a single GSM burst is approximately $577 e^{-6} \mathrm{sec}$ and the period of a Time Division Multiple Access (TDMA) frame serving 8 users is $4.614 e^{-3}$ sec. GSM used Gaussian Minimum Phase Keying (GMSK) as a modulation format. It is mainly applied in all the cellular system networks. GSM signal is mostly used in location tracking and estimating a mobile user position through cell allocation and mobile equipment coding. The point from where the signal is being transmitted can also be determined though difficult with limited packet information. However, the identified signal in

Figure 4 is a GSM signal by observation. Spectrum analyzer is an efficient tool for signal identification in the frequency domain. It aided in identifying the signal's bandwidth, frequency and other signals characteristics such as power and noise level. 


\section{CHANNEL DEMODULATION OF FREQUENCY MODULATED (FM) SIGNAL}

The channel demodulation of a 16 channels FM modulated signal which contains an AM-DSB-TC modulated signal was achieved using Figure 5. Great care was taking in ensuring that the appropriate sampling rates was used at each decimation stages of the decimation process to avoid aliasing which will result to a wrong information being decoded.

The different colours in Figure 5 shows the different frequencies used at each stage. The $2.4 \mathrm{MHz}$ information signal (red) which was down converted to $480 \mathrm{KHz}$ (green) was finally reduced to $96 \mathrm{kHz}$ (blue) at the output (speakers).

\subsection{FM Demodulation and De-multiplexing}

The data imported using the RTL-SDR tool sampled at $2.4 \mathrm{MHz}$ is fed into the decimator which reduces the sampling rate to $240 \mathrm{kHz}$ by a factor of 5, producing a complex baseband FM signal which is fed into the complex frequency discriminator, see Figure 5. The discriminator as indicated in the demodulation model is made up of delay, math function, product and complex to magnitude-angle which performs FM demodulation. The result as shown in Figure 6 is proportional to the FM multiplex sent to Finite Impulse Response (FIR) filter to bandpass filter to isolate channel 16 from other channels. The demodulated frequencies of the 16 channels are shown in Figure 7.

\subsection{Signal Recovery using Bandpass Filters}

A bandpass filter which completes the complex envelop detection is then used to recover the channel. Resampled and filtered to help remove the direct current components in the form of noise and produce a high fidelity audio signal which is at approximate sampling rate to the original signal. The three spectrum analysers were used to monitor the received signal, the demodulated multiplexed signal and the output of the filter.

The decimations carried out in the simulation was done in two stages. The first decimation configuration reduced the frequency from $2.4 \mathrm{MHz}$ to $480 \mathrm{kHz}$ while the second decimation at a factor of 5 reduce the sample rate further to audio level of $48 \mathrm{KHz}$. Both were configured as a frame based channel allowing multirate processing. The decoded signal information is affected by induced noise. The information in the audio signal could not be decoded initially because it was a mix of high frequency components of 16 different audio frequencies. The peaks at various points in Figure 6 shows the FM sidebands at their carrier frequencies having maximum value of carrier power. Frequency analyzer shown in Figure 7 indicates that channel 16 has been correctly received. Others channels can be decoded by adjusting the parameters of the model correctly. It is also very important to say that the observed audio signals were not crystal clear as a result of band limit of $5 \mathrm{kHz}$ between channels and noise due to spectrum analyzers.

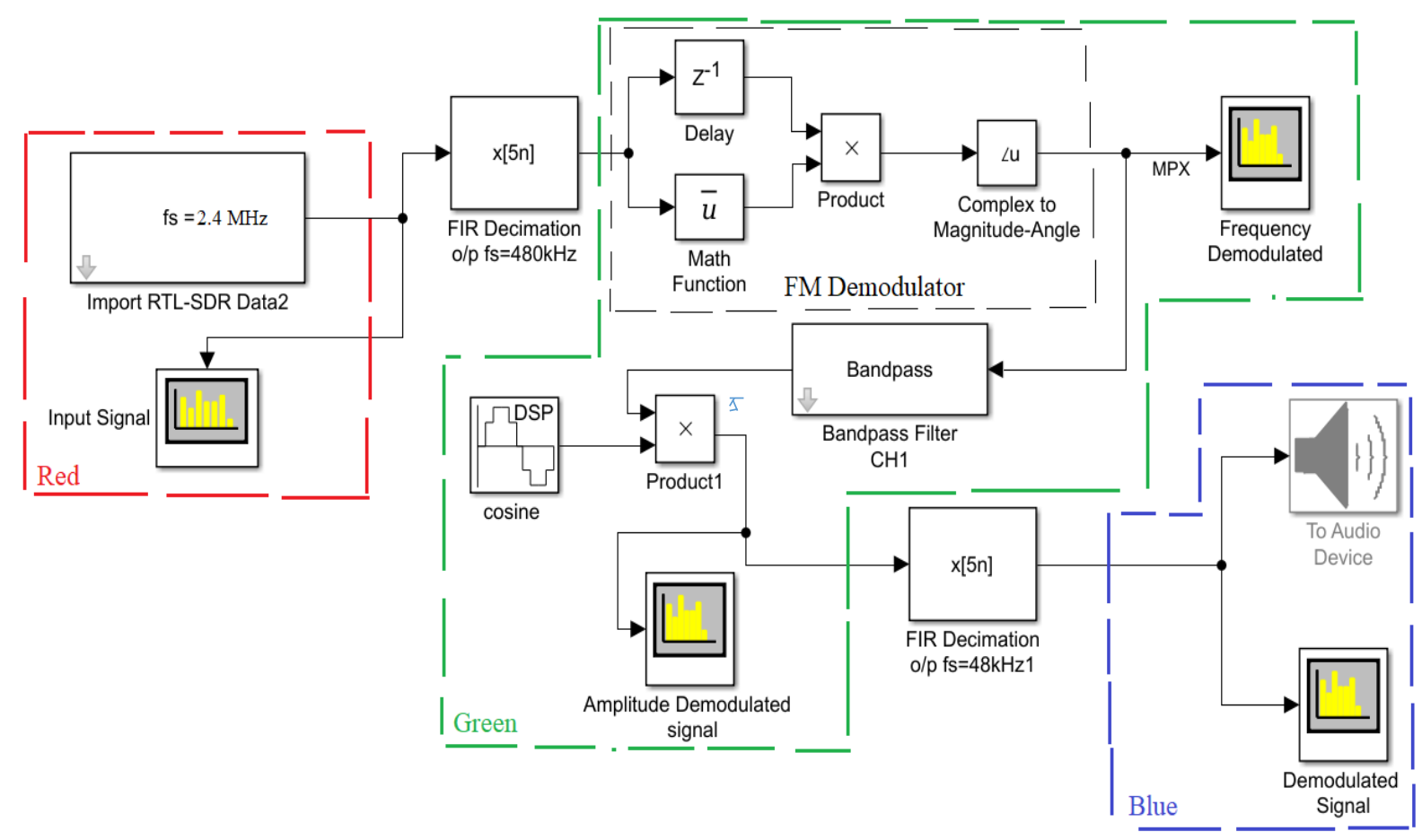

Figure 5: Demodulation of AM in FM 


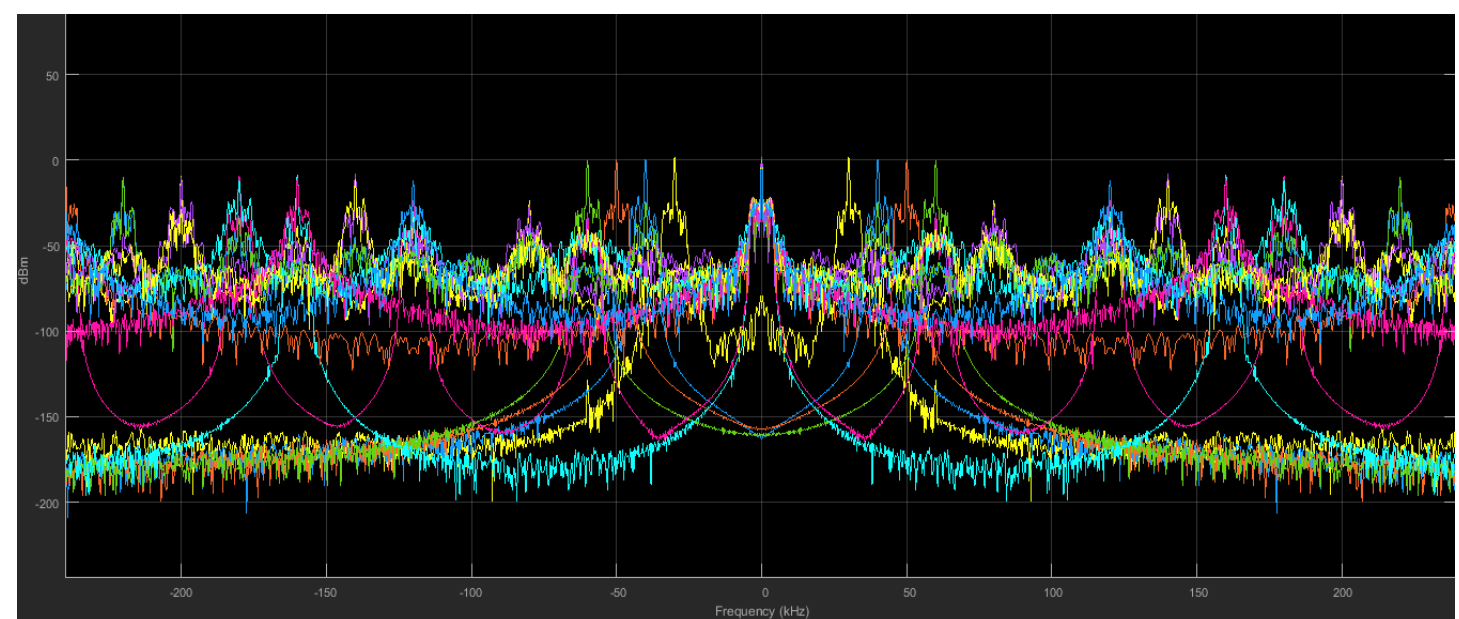

Figure 6: Demodulated Frequencies of 16 Channel Signa

\subsection{Amplitude Modulation of an Audio Signal}

The modulation of a given audio signal onto the decoded channel above is realized at $80 \mathrm{KHz}$, representing the centre frequency of the channel. Figure 8 was used for this analysis.

The input to the AM modulation model is the DSP multimedia block used to import the given audio signal. The signal interpolated using FIR filter at a factor of 4 is presented in Figure 9.

The mono 48KHz, 16-bit audio signal, having a 1024 samples per audio channel and of sample based data type was interpolated using FIR filter. The FIR resamples the signal and performs lowpass filtering thereby preventing aliasing of higher frequency components. The cosine function introduced generated the carrier signal. It is configured to have the same sample time and frequency with the modulating signal. This is to ensure that there is no phase shift between the two signals. The mathematical implication of this is shown in Figure 10 and eq. 1.

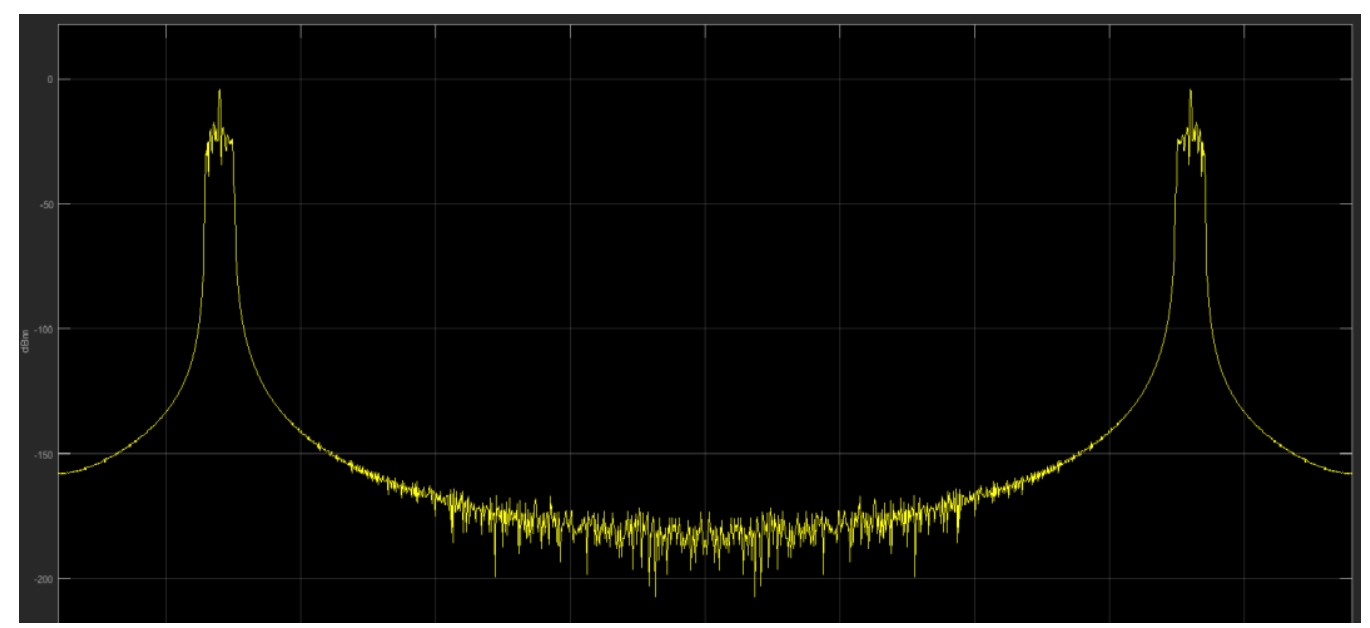

Figure 7: Demodulated Single Frequency of the 16 Channel Signal Interpolated

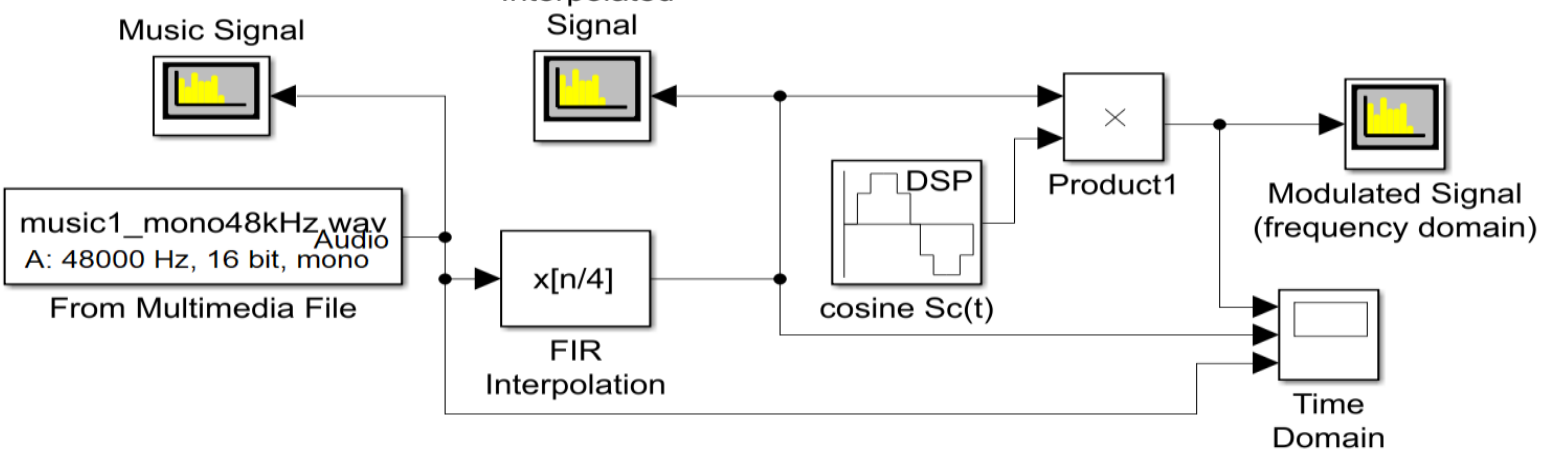

Figure 8: Amplitude Modulation Model Design 


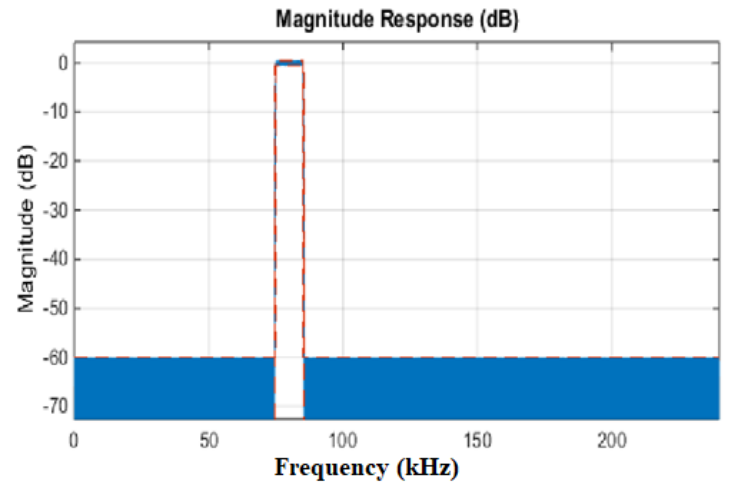

Figure 9: Interpolated Signal

\subsection{Amplitude Modulation (AM-DSB-SC)}

To amplitude modulate the given signal $S_{6}(\mathrm{t})$, it is superimposed on a carrier signal $S_{C}(\mathrm{t})$, to produce the AM signal $S_{A M-D S B-S C}(\mathrm{t}) \cdot S_{C}(\mathrm{t})$ is the frequency of the local oscillator usually made of crystal.

$$
\begin{gathered}
S_{A M-D S B-S C}(t)=S_{6}(t){ }^{*} S_{c}(t) \\
S_{6}(t)=A_{6} \operatorname{COS}\left(2 \pi f_{5} t\right) \ldots \ldots \ldots \ldots \ldots \bmod \text { ulating } \\
S_{c}(t)=A_{c} \operatorname{COS}\left(2 \pi \mathrm{f}_{\mathrm{c}} \mathrm{t}\right) \ldots \ldots \ldots \ldots \operatorname{carrier} \\
S_{A M-D S B-S C}(t)=A_{6} \operatorname{COS}\left(2 \pi f_{S} t\right){ }^{*} A_{c} \operatorname{CoS}\left(2 \pi f_{c} t\right) \\
=\frac{A_{6} A_{c}}{2}\left(\operatorname{CoS}\left(w_{c}-w_{6}\right) t\right. \\
\left.+\operatorname{COS}\left(w_{c}+w_{6}\right) t\right)
\end{gathered}
$$

The results of the interpolations carried out in the modulation process are presented in Figure 10. The frequency domain of the signal is also shown in Figure 12.

The music signal was correctly modulated as shown in Figure 12 above. The music signal contains frequency components at $80 \mathrm{kHz}$ showing an AM envelop of the modulated signal with the mirror image of the signal amplitudes pairs in time and frequency domain.

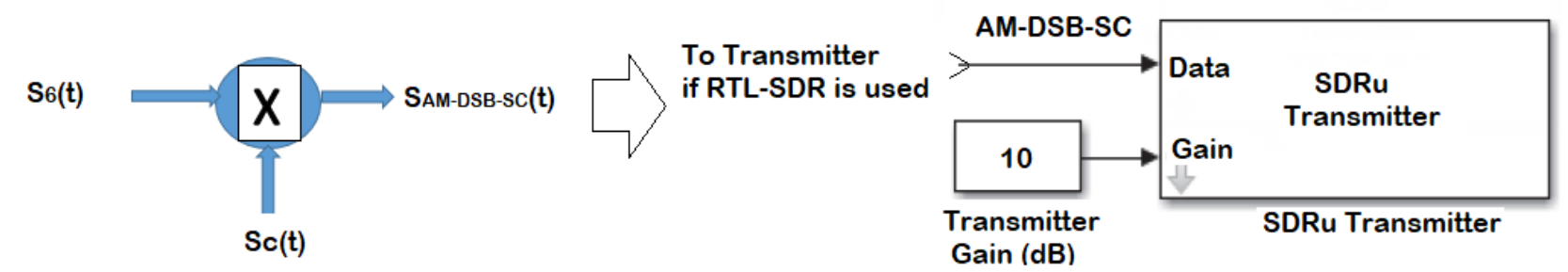

Figure 10: Audio, Decimated, and Modulated Signal

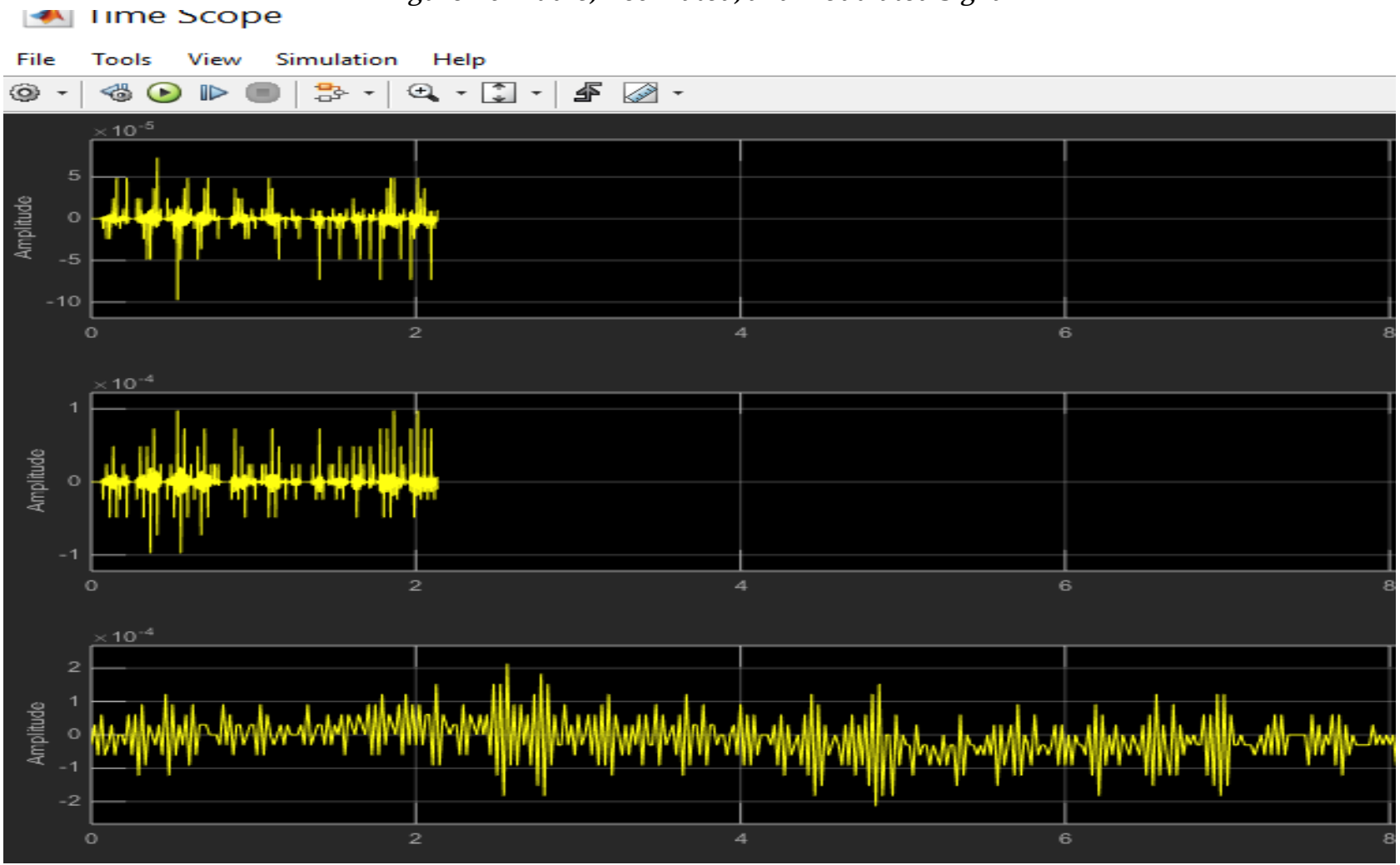

Figure 11: Time Domain of Original, Interpolated, and Modulated Signal 


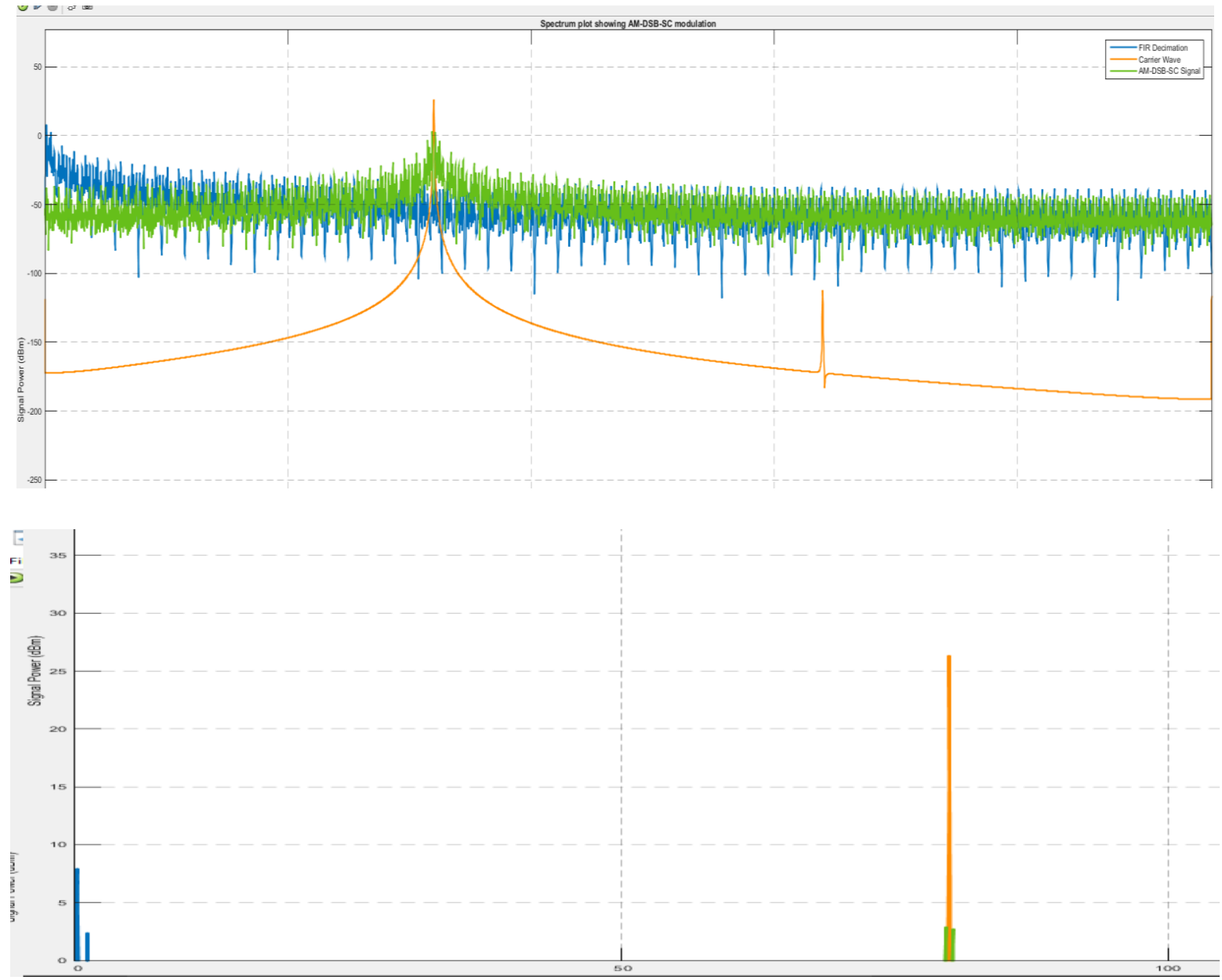

Figure 12: Frequency Domain of Original, Interpolated, and Modulated Signal

\section{THE FUTURE OF SDR}

SDR is a radio communication system in which physical layer components are implemented on a programmable platform. The signal conversions and processing is performed in software and it can support a broad range of frequencies and functions concurrently. In the ideal SDR transceiver scheme, an analog-to-digital converter (ADC) and a digital-to-analog converter (DAC) are attached to the antenna [2]. This would imply that a digital signal processor (DSP) tool is connected to the $\mathrm{ADC}$ and the $\mathrm{DAC}$, directly performing signal processing for the streams of data from/to antenna.

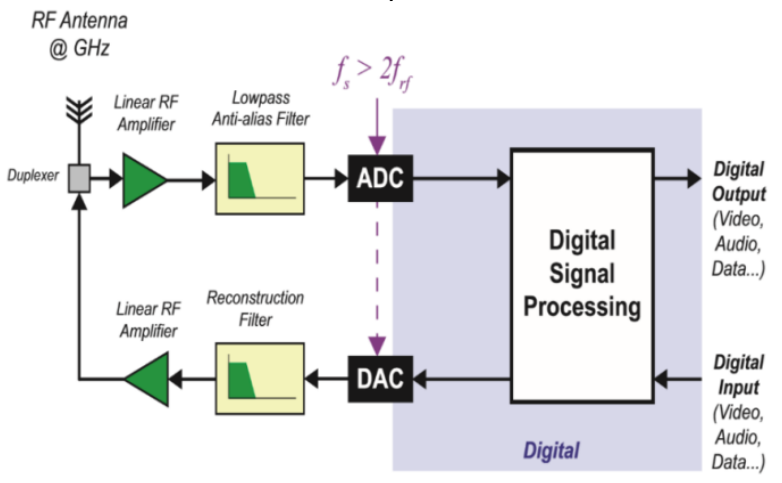

Figure 13: SDR Transceiver [9]
The benefit of moving to SDR according to [1] includes system flexibility, upgradability, customization, and adaptability; the common factors contributing to the general acceptance of this technology. The most common form of radio communication is the FM. It has wide application in commercial broadcasting services because of its high resilience to additive noise. FM is a modulation scheme that allows the variation of the signal frequency while the amplitude is kept constant. This is quite different from Amplitude Modulation (AM) where the amplitude of the signal is varied and the frequency remains unchanged. The interesting part of this study is the understanding of the theory and mathematical aspects of FM modulation and the design of receivers that can demodulate the FM signals within any location with the help of RTL-SDR device. Software Defined Radio is seen among the important components of next generation wireless communication systems because of its features not limited to sensing and spectrum monitoring, fast switching applications between many communication standards. This concept as demonstrated by the FM SDR design will improve the precision and complexity of the fundamental communications and functionalities 
of similar designs. The results of the design; a low computational complexity and high precision signal processing model and algorithm operating on digital signal processing tool boxes is presented.

The two-stage demodulation process employed in SDR are RF to IF (analogue) and IF to baseband signal (digital). These processes can be controlled through the selection of appropriate $\mathrm{RF}$ frequencies in the MATLAB Simulink toolboxes with respect to the RTL frequencies, see Figure 14.
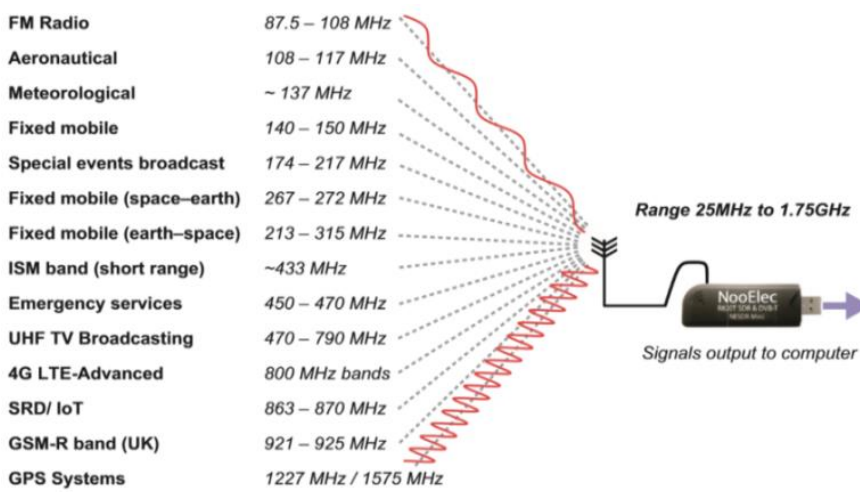

Figure 14: SDR-RTL Signals (Location Dependent) [9]

\section{CONCLUSION}

In this paper, we have explored and demonstrated the use of RTL-SDR in MATLAB and Simulink to receive and process FM, AM, and speech signal. The signal processing schemes includes modulation and demodulation, multiplexing and de-multiplexing, filtering, sampling, channel coding, decimation, interpolation, signal conversion and spectrum analysis. We have shown how FFT and waterfall analysers were used to effectively determine signal characteristics such as bandwidth, frequencies, power, noise level, and types. We also showed the mathematical and configurations issues in AM and FM modulation and demodulation, and speech signals.

The future benefits of SDR technology as shown in the last section has shown that SDR is an important part of new generation communication systems like 5G systems and internet of things based applications. There is a high demand for SDR based solution which is now driving a further investigation into the security concerns with SDR and RTL based communication systems.

\section{REFERENCES}

[1] W. H. Tuttlebee, "Software-Defined Radio: Facets of a Developing Technology," IEEE Personal Communication, Vol. 6, Issue. 2, April, pp. 38-44, 1999.

[2] R. W. Stewart, L. Crockett, D. Atkinson et al, "A Low-Cost Desktop Software Defined Radio Design Environment Using MATLAB, Simulink, and the RTL-SDR," IEEE Communication Magazine, Vol. 53, Issue. 9, September, pp. 64-71, 2015.

[3] H. Hassan, C. Ku, H. Che et al, "Low Complexity SDR Transceiver Design using Simulink, Matlab and Xilinx," IEEE International Conference on ICT Convergence (ICTC), Jeju Island, South Korea Oct. 15-17, pp. 55-60, 2012

[4] D. Sinha, A. K. Verma, and S. Kumar, "Software Defined Radio: Operation, Challenges and Possible Solutions," Intelligent Systems and Control (ISCO) 10th International Conference, Coimbatore, India, Jan. 7-8, 2016, pp. 1-5.

[5] J. Melby, "JTRS and the Evolution toward Software-Defined Radio," MILCOM Proceedings, Vol. 2, 2002, pp. 1286-1290.

[6] K. Moessner and S. Truelove, "Evaluation of Software Defined Radio Technology" www.pdfs.semanticscholar.org/143d/ecb9dc095 770ee0fcac57eb373bd6366fae7.pdf, Accessed on June 28, 2017.

[7] M. Palkovic, P. Raghavan, M. Li et al, "Future Software-Defined Radio Platforms and Mapping Flows," IEEE Signal Processing Magazine, vol. 27, Issue. 2, March, pp. 22-33, 2010.

[8] A. Sriram, A. S. R. Monalisa, and S. Sridevi, "Reconfigurable Channel Filtering Architecture Based on Two bit Sub Expression Minimization for Digital up Conversion in Software Defined Radio," 2017 International Conference on Wireless Communications, Signal Processing and Networking (WiSPNET), Chennai, India, March 22-24, 2017, pp. 1974-1978.

[9] R. W. Stewart, K. W. Barlee, D. S. W Atkinson and L. H. Crocket, Sofware-defined-radio using Matlab and Similink and the RTL-SDR, published by the University of Stacklyde Academic Media, 2015. 